\title{
Use of polysomnography with synchronized digital video recording to diagnose pediatric sleep breathing disorders
}

CASE 1: A 16-year-old male student with a body mass index (BMI) of $33.2 \mathrm{~kg} / \mathrm{m}^{2}$ was referred for evaluation because of a 1-year history of snoring, observed apnea, nocturnal restlessness and morning headache. The patient reported usually going to bed at 10:30 pm, taking 2 hours to fall asleep and awakening at $7 \mathrm{am}$. He often had to fight falling asleep in the daytime. He denied hypnagogic hallucinations (hallucinations occurring at onset of sleep), cataplexy, sleep paralysis or symptoms of restless legs. However, his mother described him as fidgety and frequently having to tap his legs. $\mathrm{He}$ was taking omeprazole, $10 \mathrm{mg} / \mathrm{d}$, because of nocturnal gastroesophageal reflux. There was no significant family history of sleep disorders. Physical examination revealed a crowded pharyngeal airway because of obesity, tonsils within normal limits and micrognathia (a small jaw). He was receiving orthodontic treatment.

The patient underwent comprehensive polysomnography with synchronized digital video recording. The apnea hypopnea index was normal, at 0.6 per hour (normal $<1.0$ per hour in children). However, there were increased respiratory efforts followed by arousals and elevated endtidal carbon dioxide levels, and the digital video showed that he snored loudly with his mouth open (Fig. 1). He also had repetitive arousals and then moved his head to re-establish breathing when on his back (see video at www.cmaj.ca/cgi/content/full/173/1/28/DC1). The arousal index was 16.1 per hour (normal $<5$ per hour). He had normal distribution of sleep stages except for reduction in the amount of rapid-eye-movement (REM) sleep (stage 1: $2.1 \%$, stage 2: $48.7 \%$, stage $3: 8.8 \%$, stage 4: $28.6 \%$, REM sleep: $11.9 \%$ [normal $20 \%-25 \%$ ]). The percutaneous arterial oxygen saturation $\left(\mathrm{SpO}_{2}\right)$ was $96.8 \%$ on average and never went below $90 \%$. The highest end-tidal $\mathrm{CO}_{2}$ level was $52.3 \mathrm{~mm} \mathrm{Hg}$, which is greater than normal. There were 8.4 leg movements per hour of sleep (normal $<5$ per hour), which were also visible on the video. His sleep study findings were consistent with pediatric obstructive sleep apnea syndrome or upper airway resistance syndrome. The patient was assessed on nasal continuous positive airway pressure (CPAP). A subsequent video recording

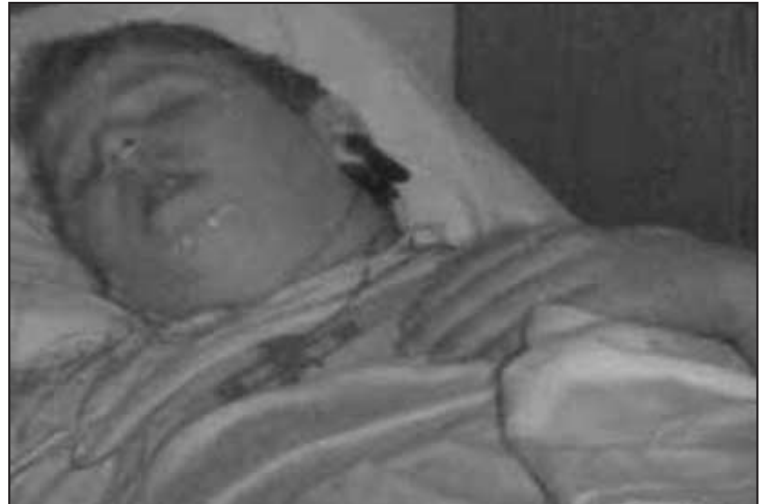

Fig. 1: Breathing through the mouth while sleeping, as seen here in case 1 , results in a posterior movement of the mandible, which may play a role in narrowing the airway behind the tongue. [Video clips of cases 1 and 2 are available online at www.cmaj.ca/cgi/content/full /173/1/28/DC1.] showed that his loud snoring and abnormal respiratory efforts were abolished. His sleep structure improved, with a decrease in arousal index from 16.1 to 9.4 per hour, and his distribution of sleep stages returned to within normal limits. The highest end-tidal $\mathrm{CO}_{2}$ level decreased from 52.3 to $47.4 \mathrm{~mm} \mathrm{Hg}$ on CPAP. The patient stated that he was more alert after treatment. A clinical trial of nasal CPAP with a pressure of $7 \mathrm{~cm} \mathrm{H}_{2} \mathrm{O}$ was started.

Hematologic evaluation, performed to rule out biochemical causes of increased restlessness, revealed a normal hemoglobin level but slightly reduced iron stores (serum ferritin level 18 [normal 20-300] $\mu \mathrm{g} / \mathrm{L}$; serum iron 11.2 [normal 7.0-27.0] $\mathrm{mmol} / \mathrm{L}$; total iron-binding capacity 62.1 [normal 47-72] $\mu \mathrm{mol} / \mathrm{L}$; \% iron saturation $18 \%$ [normal 11\%-46\%].

CASE 2: A 13-year-old male student with a BMI of $19.3 \mathrm{~kg} / \mathrm{m}^{2}$ was referred because of subjective daytime sleepiness. Although apnea was not observed by his parents, he had snored since his early years of life. His school teachers and his parents noticed that he was sleepy during the day. The patient stated that he normally went to bed between 10:00 and 10:30 pm, that it took him 30 minutes to fall asleep and that he woke up at 7:00 am. He denied hypnagogic hallucinations, cataplexy or sleep paralysis. He had symptoms of restless legs syndrome. He was taking venlafaxine, $112 \mathrm{mg} / \mathrm{d}$, and methylphenidate hydrochloride, $54 \mathrm{mg} / \mathrm{d}$, prescribed 3 years earlier for attention-deficit hyperactivity disorder (ADHD). There was no significant family history of sleep disorders. Physical ex- 
amination revealed marked retrognathia (posteriorly located lower jaw), micrognathia and nasal congestion. There were no abnormalities in the pharynx. The patient did not eat meat because the micrognathia prevented him from chewing properly.

A sleep study revealed findings consistent with pediatric obstructive sleep apnea syndrome. The apnea hypopnea index was 13.5 per hour. The $\mathrm{SpO}_{2}$ was $97.0 \%$ on average; $0.1 \%$ of the time it was below $90 \%$. The digital video recording showed that the patient had moderate snoring with snorts (see video at www.cmaj.ca/cgi/content/full/173/1/28/DC1). These were associated with $1 \%-2 \%$ oxygen desaturations that were followed by arousals detected on an electroencephalogram. His head and neck assumed a posture in which he extended his neck and protruded his lower jaw to open the airway (Fig. 2). The arousal index was 7.3 per hour. The distribution of sleep stages was within normal limits except for a shortened REM stage (stage 1: $4.8 \%$, stage 2: $58.8 \%$, stage 3: $12.2 \%$, stage $4: 16.3 \%$, REM sleep: $7.9 \%)$. The highest end-tidal $\mathrm{CO}_{2}$ level was $52.5 \mathrm{~mm} \mathrm{Hg}$. The patient demonstrated abnormal leg movements (11.3 per hour), which were visible on the video. He was not assessed on nasal CPAP because he was very thin and the obstructive sleep apnea syndrome was primarily considered to be caused by craniofacial abnormality. We recommended orthodontic treatment for his marked retrognathia.

Hematologic evaluation showed that the patient was not anemic (hemoglobin 142 [normal 125-142] g/L but that he had reduced iron stores (serum ferritin level $12 \mu \mathrm{g} / \mathrm{L}$, serum iron $17.9 \mathrm{mmol} / \mathrm{L}$, total iron-binding capacity $71.7 \mu \mathrm{mol} / \mathrm{L}$ and \% iron saturation $25 \%$ ).

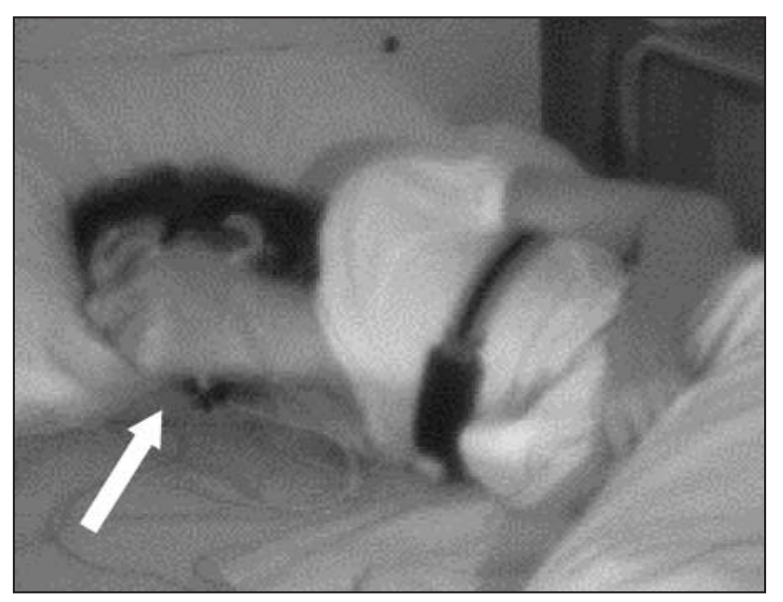

Fig. 2: Abnormal extension of neck and protrusion of jaw (arrow) to open the airway in case 2.
$\mathrm{T}$ he prevalence of sleep breathing disorders in a pediatric population has been reported to range from $0.7 \%$ to $10.3 \% .^{1}$ These disorders encompass a wide spectrum of sleeprelated abnormalities, from obstructive sleep apnea syndrome to upper airway resistance syndrome to obesity-related hypoventilation. Obstructive sleep apnea syndrome is a common type of sleep breathing disorder and is characterized by snoring, observed apnea and daytime sleepiness. In pediatric cases, this syndrome can cause several important sequelae, including growth retardation, cardiovascular comorbidities and neurobehavioural problems. The diagnostic criteria for sleep breathing disorders in children and adolescents differ from those in adults. An apnea hypopnea index of more than 1 per hour is often chosen as the cutoff for normality. ${ }^{1}$ In pediatric cases, apnea is defined as absence of airflow at the nose and mouth for more than 2 breaths and hypopnea as reduction of at least $50 \%$ in oronasal flow signal amplitude for more than 2 breaths. Some pediatric cases may have a sleep breathing disorder even though polysomnographic findings do not meet the diagnostic criteria. The diagnosis in pediatric cases may be overlooked if comprehensive polysomnography is used without synchronized video recording.

Upper airway resistance syndrome, a subtle form of obstructive sleep apnea syndrome, is characterized by increased upper airway resistance during sleep and leads to increased respiratory effort and sleep fragmentation, and subjective daytime sleepiness. The apnea hypopnea index is within normal limits in such cases. Treatment of this syndrome is often with an oral appliance or CPAP. To establish a diagnosis, continuous nocturnal monitoring of esophageal pressure to measure arousals related to respiratory effort is sometimes recommended. This form of monitoring, which is not routinely done during polysomnography, may be invasive to patients, especially children. However, our sleep study and video recording in case 1 showed objective audiovisual evidence of arousals caused by respiratory efforts with repetitive head movements to re-establish breathing. These events were consistent with clinical findings of upper airway resistance syndrome. We believe that video recording is more acceptable than esophageal pressure monitoring in pediatric cases of suspected sleep breathing disorders.

In the 2 cases we have described, both patients underwent comprehensive polysomnography supplemented by synchronized digital video recordings. The comprehensive polysomnography included monitoring of neuro- 
physiologic measures (electroencephalography, chin and anterior tibialis electromyelography, and electro-olfactography) and of cardiorespiratory variables (chest wall motion, abdominal motion, nasal pressure, oronasal $\mathrm{CO}_{2}$ level, $\mathrm{SpO}_{2}$ [by ear oximeter] and electrocardiography). In case 1, the synchronized video recording helped to confirm the diagnosis because there was insufficient information from the polysomnographic readings, and in case 2 it yielded the information needed to recommend treatment.

In case 1, although the number of apnea and hypopnea episodes per hour of sleep was within normal limits, the video recording showed clear evidence of abnormal respiratory efforts (movements of the head) followed by arousals. Nocturnal sleep breathing and sleep structure were improved with the use of CPAP. According to the recommended normal polysomnographic respiratory values in pediatric cases, ${ }^{2}$ only the end-tidal $\mathrm{CO}_{2}$ level was abnormal in case 1 . It has been reported that current polysomnographic criteria for pediatric cases may be inadequate for identifying breathing abnormalities during sleep. ${ }^{3}$ Comprehensive polysomnography without synchronized video recording would have missed the diagnostic findings in case 1 .

In case 2, synchronized video data demonstrated snorts that opened the upper airway after oxygen saturation dropped slightly; it also revealed that the patient often aroused to extend his neck backward and protrude the lower jaw forward to open the airway. These events captured on video provided information to support the recommendation for orthodontic treatment.

Both patients had micrognathia on physical examination. The cause of sleep breathing disorder was thought to be a combination of obesity and micrognathia in case 1 and micrognathia in case 2.

Both cases had demonstrated abnormal movements, which were recorded on the videos and on anterior tibialis electromyelography. Although they did not have anemia, both children had reduced iron stores. Low body stores of iron may cause restless leg syndrome, which results in insomnia in some teenagers. ${ }^{4}$ Case 2 had ADHD, and sleep disorders may be related to this condition. Because sleep disorders in children may result in symptoms that overlap those of ADHD, appropriate diagnostic techniques and treatment of the sleep disorder may eliminate the symptoms suggestive of ADHD.

In summary, synchronized digital video recording yields clear audiovisual information of abnormal nocturnal breathing that is not detected with the use of standard polysomnography. Therefore, we recommend its use in the assessment of suspected cases of pediatric sleep breathing disorders.

\section{Katsuhisa Banno}

Meir H. Kryger

Sleep Disorders Centre

St. Boniface General Hospital

University of Manitoba

Winnipeg, Man.

This article has been peer reviewed.

Acknowledgement: Our work is supported by grant R01 HL63342-01A1 from the National Institutes of Health.

\section{References}

1. Schechter MS; Section on Pediatric Pulmonology, Subcommittee on $\mathrm{Ob}$ structive Sleep Apnea Syndrome. Technical report: diagnosis and management of childhood obstructive sleep apnea syndrome. Pediatrics 2002;109(4):e69.

2. Uliel S, Tauman R, Greenfeld M, Sivan Y. Normal polysomnographic respiratory values in children and adolescents. Chest 2004;125(3):872-8.

3. Guilleminault C, Li K, Khramtsov A, Palombini L, Pelayo R. Breathing patterns in prepubertal children with sleep-related breathing disorders. Arch Pediatr Adolesc Med 2004;158(2):153-61.

4. Kryger MH, Otake K, Foerster J. Low body stores of iron and restless legs syndrome: a correctable cause of insomnia in adolescents and teenagers. Sleep Med 2002;3(2):127-32 